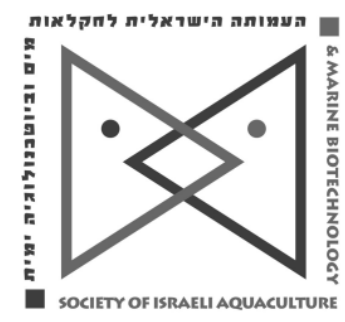

The IJA appears exclusively as a peer-reviewed on-line open access journal at http://www.siamb.org.il

Sale of IJA papers is strictly forbidden.

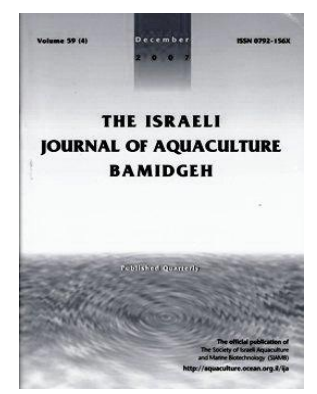

\title{
Effects of L-Carnitine on Growth of Individually Cultured Cladoceran, Moina micrura
}

\author{
S. Savas*, O. Erdogan, N.L. Cicek
}

Süleyman Demirel University, Faculty of Fisheries, Eğirdir, Isparta, Turkey

(Received 30.7.10, Accepted 22.9.10)

Key words: Moina micrura, L-carnitine, population growth

\begin{abstract}
The cladoceran, Moina micrura, is a potential alternative live food for fish larvae. The effects of L-carnitine enrichment on first reproduction age, population density, and growth rate in $M$. micrura were determined. The cladocerans were enriched at 10 levels of L-carnitine $(0,0.001,0.01,0.1,1$, $10,100,500,1000$, and $1500 \mathrm{mg} / \mathrm{l}$ ) dissolved in $30 \mathrm{ml}$ Chlorella vulgaris culture medium for 17 days. The first reproduction age was stimulated by the supplemental L-carnitine. The population density was highest in the $1 \mathrm{mg} / \mathrm{l}$ treatment. The highest population growth rate was obtained in the $10 \mathrm{mg} / \mathrm{l}$ treatment, but it did not differ from the growth rate in the $0.1,1$, or $100 \mathrm{mg} / \mathrm{l}$ treatments. Consequently, the optimum L-carnitine concentration for $M$. micrura is $1 \mathrm{mg} / \mathrm{l}$. Results suggest that L-carnitine can be a positive factor in enhancing the use of $M$. micrura as an alternative live food for fish larvae.
\end{abstract}

\footnotetext{
* Corresponding author. Tel.:+90-246-3133447, fax: +90-246-3133452, e-mail:
} segi68@yahoo.com 


\section{Introduction}

Food and feeding regimes are important factors in zooplankton cultures such as rotifer and daphnia. Knowledge of the combined impacts of these two factors is useful for developing efficient aquaculture practices. One the most important variables affected by food quality is the population growth rate. The effects of food on the dynamics of cladocerans have been well-researched (Nandini and Sarma, 2003; Alva-Martinez et al., 2004) but little information is available on the effect of different foods on Moina micrura (Jana and Pal, 1985; Ovie and Egborge, 2002; Pagano, 2008).

The vitamin-like nutrient, L-carnitine, has long attracted researchers because of its important function in fostering oxidation by mitochondria of long-chain fatty acids and stimulating protein-sparing action by increasing energy derived from lipids (Harpaz, 2005). This study investigated the effects of L-carnitine supplementation on the reproduction age, population density, and population growth of the cladoceran, Moina micrura.

\section{Materials and Methods}

Moina micrura were isolated from Lake Titreyen in Antalya, Turkey, and maintained at least three years in laboratory conditions. The $M$. micrura were first enhanced with 10 levels of L-carnitine $(0,0.001,0.01,0.1,1.0,10,100,500,1000$, and $1500 \mathrm{mg} / \mathrm{l})$, dissolved in Chlorella vulgaris culture medium. When the cladocerans adapted to the Lcarnitine level, neonatal $M$. micrura less than 24 hours old were enhanced a second time. Five M. micrura were individually pipetted into a culture vessel containing $30 \mathrm{ml}$ sterile medium enriched with the same levels of L-carnitine as above. For each concentration, three replicate wells were prepared. Trials were conducted in a C. vulgaris density of $1.5 \mathrm{x}$ $10^{6}$ cells $/ \mathrm{ml}$ at $25 \pm 1^{\circ} \mathrm{C}$ under axenic conditions for 17 days, with a photoperiod of 16 light: 8 dark hours. The number of individuals in each population was determined every $24 \mathrm{~h}$. The culture medium was renewed every $48 \mathrm{~h}$ and kept at the same cell density. The rate of population increase was determined as ( In $\left.N_{t}-\ln N_{0}\right) / t$, where $N_{0}=$ initial population density and $\mathrm{N}_{\mathrm{t}}=$ population density at time $\mathrm{t}$ (Nandini and Sarma, 2003).

Statistical analyses were performed using SPSS 12.0 for Windows software (SPSS Inc, Chicago, IL). Differences between treatments in population density and population growth rate were tested using ANOVA. Data were analyzed by one-way analysis of variance (ANOVA) and multiple comparisons were made by Duncan's multiple range test. The significance level was $p<0.05$.

\section{Results}

At the end of the 17 days, the population density was significantly higher in all enriched groups than in the unsupplemented control (Table 1). The highest population growth rate was obtained in the $10 \mathrm{mg} / \mathrm{l}$ treatment (Table 2). Enrichment with $500 \mathrm{mg} / \mathrm{l} \mathrm{L}$-carnitine caused $100 \%$ mortality within five days of culture while enrichment with 1000 and 1500 $\mathrm{mg} / \mathrm{l}$ caused $100 \%$ mortality rate on the first day. The reproduction age in the treatments significantly differed from that of the control.

\section{Discussion}

Manipulation of nutrition and growth to produce greater quantities of and better quality live food is important in aquaculture. Population density was highest in the 0.1 and 1.0 $\mathrm{mg} / \mathrm{l}$ groups. The highest population growth rate was observed in the $10 \mathrm{mg} / \mathrm{l}$ treatment, but it did not significantly differ from the $0.1,1$, or $100 \mathrm{mg} / \mathrm{l}$ groups. Thus, the optimum concentration of L-carnitine in the culture medium is $1 \mathrm{mg} / \mathrm{l}$.

The most important environmental factors controlling cladoceran growth and reproduction are temperature, food quantity, and food quality. By virtue of their small size and short reproductive cycle, cladocerans respond rapidly to changes in food quality (Nandini and Sarma, 2003). A rapid increase in population occurs under the favorable conditions provided by an abundance of food. L-carnitine enrichment affects the population growth, egg ratio, and body size of the marine rotifer, Brachionus rotundiformis, where, in individual culture trials, the population densities between 
Table 1. Population density (individuals/30 ml) of Moina micrura enriched with different levels of L-carnitine.

\begin{tabular}{|c|c|c|c|c|c|c|c|}
\hline \multirow{2}{*}{ Day } & \multicolumn{7}{|c|}{ L-carnitine enrichment level } \\
\hline & 0 & $0.001 \mathrm{mg} / \mathrm{l}$ & $0.01 \mathrm{mg} / \mathrm{l}$ & $0.1 \mathrm{mg} / \mathrm{l}$ & $1 \mathrm{mg} / \mathrm{l}$ & $10 \mathrm{mg} / \mathrm{I}$ & $100 \mathrm{mg} / \mathrm{l}$ \\
\hline 1 & $5.00 \pm 0.00$ & $5.00 \pm 0.00$ & $5.00 \pm 0.00$ & $5.00 \pm 0.00$ & $5.00 \pm 0.00$ & $5.00 \pm 0.00$ & $5.00 \pm 0.00$ \\
\hline 2 & $5.00 \pm 0.00$ & $5.00 \pm 0.00$ & $5.00 \pm 0.00$ & $5.00 \pm 0.00$ & $5.00 \pm 0.00$ & $5.00 \pm 0.00$ & $5.00 \pm 0.00$ \\
\hline 3 & $5.00 \pm 0.00$ & $5.00 \pm 0.00$ & $5.00 \pm 0.00$ & $5.00 \pm 0.00$ & $5.00 \pm 0.00$ & $5.00 \pm 0.00$ & $5.00 \pm 0.00$ \\
\hline 4 & $5.00 \pm 0.00^{b}$ & $7.33 \pm 2.33^{b}$ & $8.67 \pm 3.67^{b}$ & $8.00 \pm 1.73^{b}$ & $18.33 \pm 4.70^{a}$ & $13.67 \pm 1.76^{\mathrm{ab}}$ & $6.67 \pm 1.67^{b}$ \\
\hline 5 & $11.67 \pm 3.38^{c}$ & $12.67 \pm 4.10^{\mathrm{bc}}$ & $22.00 \pm 3.46^{\mathrm{ab}}$ & $20.00 \pm 1.53^{\mathrm{abc}}$ & $25.00 \pm 2.65^{a}$ & $23.33 \pm 2.85^{a}$ & $12.67 \pm 2.91^{\mathrm{bc}}$ \\
\hline 6 & $17.33 \pm 3.93^{c}$ & $28.00 \pm 5.13^{b c}$ & $32.00 \pm 2.65^{\mathrm{b}}$ & $47.00 \pm 4.16^{\mathrm{a}}$ & $39.33 \pm 3.38^{\mathrm{ab}}$ & $30.00 \pm 3.21^{b c}$ & $29.00 \pm 5.51^{b c}$ \\
\hline 7 & $32.33 \pm 4.63^{c}$ & $41.00 \pm 11.79^{\mathrm{bc}}$ & $45.00 \pm 5.57^{\mathrm{bc}}$ & $90.00 \pm 4.51^{a}$ & $62.33 \pm 6.49^{b}$ & $49.33 \pm 0.67^{\mathrm{bc}}$ & $54.67 \pm 5.84^{b}$ \\
\hline 8 & $41.67 \pm 3.33^{c}$ & $65.67 \pm 15.34^{b c}$ & $58.67 \pm 3.48^{\mathrm{bc}}$ & $94.67 \pm 3.33^{\mathrm{a}}$ & $77.67 \pm 3.84^{\mathrm{ab}}$ & $85.67 \pm 4.33^{\mathrm{ab}}$ & $74.67 \pm 13.62^{a b}$ \\
\hline 9 & $54.00 \pm 3.21^{d}$ & $86.00 \pm 11.14^{c}$ & $119.67 \pm 9.17^{\mathrm{ab}}$ & $119.33 \pm 2.03^{\mathrm{ab}}$ & $99.00 \pm 11.59^{\mathrm{abc}}$ & $126.33 \pm 2.96^{\mathrm{a}}$ & $92.00 \pm 16.70^{b c}$ \\
\hline 10 & $65.67 \pm 8.09^{d}$ & $102.33 \pm 12.20^{c}$ & $148.67 \pm 5.78^{\mathrm{ab}}$ & $124.67 \pm 6.64^{b c}$ & $126.33 \pm 2.33^{b c}$ & $170.67 \pm 8.17^{a}$ & $148.33 \pm 16.02^{\mathrm{ab}}$ \\
\hline 11 & $84.33 \pm 0.88^{d}$ & $121.33 \pm 9.53^{c}$ & $169.00 \pm 9.07^{b}$ & $178.00 \pm 5.29^{b}$ & $195.67 \pm 7.88^{b}$ & $243.67 \pm 15.07^{a}$ & $199.67 \pm 22.21^{b}$ \\
\hline 12 & $105.33 \pm 6.77^{e}$ & $144.33 \pm 6.74^{d}$ & $190.33 \pm 10.59^{c}$ & $229.33 \pm 5.81^{b c}$ & $240.67 \pm 1.76^{b}$ & $287.00 \pm 4.36^{\mathrm{a}}$ & $215.67 \pm 28.54^{\mathrm{bc}}$ \\
\hline 13 & $111.67 \pm 5.55^{\mathrm{e}}$ & $153.33 \pm 7.42^{d}$ & $219.00 \pm 0.58^{c}$ & $266.33 \pm 5.24^{b}$ & $302.33 \pm 4.33^{a}$ & $241.67 \pm 4.91^{c}$ & $177.00 \pm 17.39^{d}$ \\
\hline 14 & $94.33 \pm 8.84^{d}$ & $170.00 \pm 14.05^{c}$ & $208.67 \pm 11.98^{b}$ & $268.00 \pm 8.72^{a}$ & $280.00 \pm 3.46^{a}$ & $215.67 \pm 6.98^{b}$ & $163.67 \pm 13.69^{c}$ \\
\hline 15 & $75.00 \pm 9.50^{d}$ & $186.00 \pm 9.64^{b}$ & $189.67 \pm 10.37^{b}$ & $230.33 \pm 5.17^{a}$ & $232.33 \pm 6.49^{a}$ & $203.67 \pm 3.84^{b}$ & $155.00 \pm 8.66^{c}$ \\
\hline 16 & $65.00 \pm 2.89^{e}$ & $149.33 \pm 6.44^{d}$ & $177.00 \pm 4.04^{c}$ & $219.67 \pm 2.96^{a}$ & $219.33 \pm 1.20^{a}$ & $190.33 \pm 2.33^{b}$ & $149.33 \pm 6.36^{d}$ \\
\hline 17 & $62.33 \pm 1.86^{\mathrm{e}}$ & $121.00 \pm 3.79^{d}$ & $166.33 \pm 2.03^{b}$ & $209.00 \pm 2.08^{a}$ & $211.67 \pm 2.19^{a}$ & $170.00 \pm 0.58^{b}$ & $147.00 \pm 5.51^{c}$ \\
\hline
\end{tabular}

Different superscripts within a row indicate significant differences $(p<0.05)$.

Table 2. Population growth rate (per day) of Moina micrura enriched with different levels of Lcarnitine $(\mathrm{mg} / \mathrm{l})$.

\begin{tabular}{|c|c|c|c|c|c|c|c|}
\hline \multirow{2}{*}{ Day } & \multicolumn{7}{|c|}{ L-carnitine enrichment level } \\
\hline & 0 & $0.001 \mathrm{mg} / \mathrm{l}$ & $01 \mathrm{mg} / \mathrm{l}$ & $0.1 \mathrm{mg} / \mathrm{l}$ & $1 \mathrm{mg} / \mathrm{l}$ & $10 \mathrm{mg} / \mathrm{l}$ & $100 \mathrm{mg} / \mathrm{l}$ \\
\hline 1 & $0.000 \pm 0.000$ & $0.000 \pm 0.000$ & $0.000 \pm 0.000$ & $000 \pm 0.000$ & $0.000 \pm 0.000$ & $0.000 \pm 0.000$ & $0.000 \pm 0.000$ \\
\hline 2 & $0.000 \pm 0.000$ & $0.000 \pm$ & $.000 \pm 0.000$ & 0 & 00 & 00 & 000 \\
\hline 3 & $0.000 \pm c$ & .00 & 000 & 0 & 0 & 0.0 & \\
\hline 4 & $0.000 \pm 0.000^{c}$ & $0.019 \pm 0.019^{b c}$ & $0.030 \pm 0.030^{\mathrm{bc}}$ & $0.031 \pm 0.018^{\mathrm{bc}}$ & $0.093 \pm 0.024^{a}$ & $0.082 \pm 0.011^{\mathrm{ab}}$ & $0.019 \pm 0.019^{b c}$ \\
\hline 5 & $0.059 \pm 0.030^{c}$ & $0.055 \pm 0.028^{c}$ & $0.102 \pm 0.016^{\mathrm{ab}}$ & $0.103 \pm 0.005^{\mathrm{ab}}$ & $0.123 \pm 0.008^{a}$ & $0.127 \pm 0.010^{\mathrm{a}}$ & $0.075 \pm 0.020^{\mathrm{ab}}$ \\
\hline 6 & $0.094 \pm 0.016^{c}$ & $.115 \pm 0.015^{b c}$ & $0.132 \pm 0.007^{\mathrm{abc}}$ & 0.16 & $0.158 \pm 0.006^{\mathrm{a}}$ & $0.148 \pm$ & $0.148 \pm 0.021^{\mathrm{ab}}$ \\
\hline 7 & $0.146 \pm 0 . C$ & $0.138 \pm 0$. & $0.154 \pm 0.005^{\mathrm{b}}$ & $0.217 \pm 0.009^{a}$ & $0.193 \pm 0.008^{a}$ & 0.19 & $0.205 \pm$ \\
\hline 8 & $0.167 \pm 0.010^{c}$ & $0.171 \pm 0.021^{\mathrm{c}}$ & $0.183 \pm 0.008^{b c}$ & $0.221 \pm 0.005^{\mathrm{ab}}$ & $0.211 \pm 0.004^{\mathrm{abc}}$ & 0.23 & $0.230 \pm 0.022^{a}$ \\
\hline 9 & $0.188 \pm 0.008^{d}$ & $0.193 \pm 0.012^{\mathrm{cd}}$ & $0.233 \pm 0.006^{b}$ & $0.238 \pm 0.006^{\mathrm{ab}}$ & $0.229 \pm 0.009^{b c}$ & $0.269 \pm 0.002^{a}$ & $0.248 \pm 0.022^{\mathrm{ab}}$ \\
\hline 10 & $0.203 \pm 0.015^{c}$ & $0.205 \pm 0.012^{c}$ & $0.253 \pm 0.006^{b}$ & $0.241 \pm 0.007^{b}$ & $0.248 \pm 0.001^{b}$ & $0.294 \pm 0.004^{a}$ & $0.291 \pm 0.018^{\mathrm{a}}$ \\
\hline 11 & $0.223 \pm 0.006^{c}$ & $0.217 \pm 0.010^{c}$ & $0.264 \pm 0.006^{\mathrm{b}}$ & $0.268 \pm 0.007^{b}$ & $0.282=$ & $0.324 \pm 0.005^{\mathrm{a}}$ & $0.316 \pm 0.019^{a}$ \\
\hline 12 & $0.241 \pm 0.011^{d}$ & $0.230 \pm 0.008^{d}$ & $0.269 \pm 0.007^{c}$ & $0.287 \pm 0.007^{\mathrm{bc}}$ & $0.298 \pm 0.001^{b}$ & $0.338 \pm 0.001^{a}$ & $0.321 \pm 0.003^{a}$ \\
\hline 13 & $0.245 \pm 0.007^{d}$ & $0.234 \pm 0.009^{d}$ & $0.284 \pm 0.007^{c}$ & $0.299 \pm 0.009^{b c}$ & $0.316 \pm 0.001^{\mathrm{ab}}$ & $0.323 \pm 0.002^{\mathrm{a}}$ & $0.305 \pm 0.002^{\mathrm{ab}}$ \\
\hline 14 & $0.232 \pm 0.011^{c}$ & $0.241 \pm 0.011^{c}$ & $0.277 \pm 0.006^{\mathrm{b}}$ & $0.299 \pm 0.005^{\mathrm{ab}}$ & $0.310 \pm 0.001^{\mathrm{a}}$ & $0.314 \pm 0.003^{a}$ & $0.299 \pm 0.005^{\mathrm{ab}}$ \\
\hline 15 & $0.213 \pm 0.016^{d}$ & $0.247 \pm 0.003^{c}$ & $0.268 \pm 0.007^{b c}$ & $0.288 \pm 0.006^{\mathrm{ab}}$ & $0.295 \pm 0.002^{a}$ & $0.309 \pm 0.002^{a}$ & $0.294 \pm 0.005^{a}$ \\
\hline 16 & $0.203 \pm 0.009^{d}$ & $0.232 \pm 0.004^{c}$ & $0.266 \pm 0.007^{b}$ & $0.284 \pm 0.008^{a}$ & $0.291 \pm 0.000^{a}$ & $0.303 \pm 0.001^{a}$ & $0.291 \pm 0.005^{a}$ \\
\hline 17 & $0.200 \pm 0.008^{c}$ & $0.217 \pm 0.003^{c}$ & $0.262 \pm 0.007^{b}$ & $0.280 \pm 0.007^{a}$ & $0.288 \pm 0.001^{a}$ & $0.294 \pm 0.000^{a}$ & $0.290 \pm 0.006^{a}$ \\
\hline
\end{tabular}

Different superscripts within a row indicate significant differences $(p<0.05)$.

treatments significantly differed from those obtained in the present study (Zhang et al., 2005).

Supplementation of L-carnitine resulted in significant responses in reproduction and individual growth of $M$. micrura. The reproduction age differed between the treatments and the control group in the present study. Similarly, L-carnitine stimulated reproduction of enriched rotifers (Zhank et al., 2005). The population density was significantly lower in M. micrura fed only C. vulgaris. Supplementation with $500 \mathrm{mg} / \mathrm{l} \mathrm{L}$-carnitine produced $100 \%$ mortality within five days while 1000 and $1500 \mathrm{mg} / \mathrm{l}$ supplementation caused $100 \%$ mortality on the first day. Similarly, mortality was $100 \%$ within ten days in rotifers enriched with $1000 \mathrm{mg} / \mathrm{l}$ L-carnitine (Zhang et al., 2005). The highest population density ( $p=0.05$ ) was obtained in the $1 \mathrm{mg} / \mathrm{l}$ treatment after 13 days culture. The highest population growth rate was obtained in the $10 \mathrm{mg} / \mathrm{l}$ treatment, but it did not differ from 
the growth rate in the $1 \mathrm{mg} / \mathrm{l}$ treatment. Consequently, the optimum L-carnitine concentration for $M$. micrura is $1 \mathrm{mg} / \mathrm{l}$.

\section{Acknowledgements}

This study was supported by the Scientific and Technological Research Council of Turkey (TUBİTAK-1070575). The authors would like to thank Tubitak.

\section{References}

Alva-Martinez A.F., Sarma S.S.S. and S. Nandini, 2004. Population growth of Daphnia pulex (Cladocera) on a mixed diet (Microcystis aeruginosa with Chlorella or Scenedesmus). Crustaceana, 77:973-988.

Harpaz S., 2005. L-carnitine and its attributed functions in fish culture and nutrition - a review. Aquaculture, 249:3-21.

Jana B.B. and G.P. Pal, 1985. The life history parameters of Moina micrura (Kurz) grown in different culturing media. Water Res., 19(7):863-867.

Nandini S. and S.S.S. Sarma, 2003. Population growth of some genera of cladocerans in relation to algal food (Chlorella vulgaris) levels. Hydrobiologia, 491:211-219.

Ovie S.I. and A.B.M. Egborge, 2002. The effect of different algal densities of Scenedesmus acuminatus on the population growth of Moina micrura Kurz (Crustacea: Anomapoda, Moinidae). Hydrobiologia, 477(1-3):41-45.

Pagano M., 2008. Feeding of tropical cladocerans (Moina micrura, Diaphanosoma excisum) and rotifer (Brachionus calyciflorus) on natural phytoplankton: effect of phytoplankton size-structure. J. Plankton Res., 30(4):401-414.

Zhang D.M., Yoshimatsu T. and M. Furuse, 2005. Effect of L-carnitine enrichment on the population growth, egg ratio and body size of marine rotifer, Brachionus rotundiformis. Aquaculture, 248:51-57. 